\title{
Monitoring and Modelling of the Effects of Process Settings and Screw Geometry on Melt Pressure Generation in Polymer Extrusion
}

\author{
Document Version \\ Accepted author manuscript
}

Link to publication record in Manchester Research Explorer

Citation for published version (APA):

Abeykoon, C., Li, K., Martin, P. J., \& Kelly, A. L. (2012). Monitoring and Modelling of the Effects of Process Settings and Screw Geometry on Melt Pressure Generation in Polymer Extrusion. International Journal of System Control and Information Processing, 1(1), 71-88.

Published in:

International Journal of System Control and Information Processing

\section{Citing this paper}

Please note that where the full-text provided on Manchester Research Explorer is the Author Accepted Manuscript or Proof version this may differ from the final Published version. If citing, it is advised that you check and use the publisher's definitive version.

\section{General rights}

Copyright and moral rights for the publications made accessible in the Research Explorer are retained by the authors and/or other copyright owners and it is a condition of accessing publications that users recognise and abide by the legal requirements associated with these rights.

\section{Takedown policy}

If you believe that this document breaches copyright please refer to the University of Manchester's Takedown Procedures [http://man.ac.uk/04Y6Bo] or contact uml.scholarlycommunications@manchester.ac.uk providing relevant details, so we can investigate your claim.

\section{OPEN ACCESS}




\title{
Monitoring and modelling of the effects of process settings and screw geometry on melt pressure generation in polymer extrusion
}

\author{
Chamil Abeykoon* \\ School of Mechanical and Aerospace Engineering, \\ Queen's University Belfast, \\ BT9 5AH Belfast, UK \\ E-mail: yabeykoon01@qub.ac.uk \\ ${ }^{*}$ Corresponding author \\ Kang Li \\ School of Electronics, \\ Electrical Engineering and Computer Science, \\ Queen's University Belfast, \\ BT9 5AH Belfast, UK \\ E-mail: k.li@qub.ac.uk

\section{Peter J. Martin} \\ School of Mechanical and Aerospace Engineering, \\ Queen's University Belfast, \\ BT9 5AH Belfast, UK \\ E-mail: p.j.martin@qub.ac.uk
}

\author{
Adrian L. Kelly \\ IRC in Polymer Science and Technology, \\ School of Engineering, Design and Technology, \\ University of Bradford, \\ BD7 1DP Bradford, UK \\ E-mail: A.L.Kelly@bradford.ac.uk
}

\begin{abstract}
Melt pressure is one of the most important process parameters in polymer extrusion and is closely related to product quality. However, it is not directly controllable and may be affected in a complex manner by changing other process operating conditions such as screw speed and barrel set temperatures. The ability to predict such parameters would be a powerful tool to aid process design and optimisation. However, only a few practical process models are currently available to predict melt pressure based on process settings in polymer extrusion. This paper describes new non-linear static and linear dynamic models that have been developed to explore the effects of process settings and screw geometry on melt pressure development in single screw extrusion. The models developed predict the melt pressure with good accuracy over a wide operating window. Investigations made using these models together with a frequency analysis of
\end{abstract}


the measured signals showed that the melt pressure is influenced by both process settings and screw geometry.

Keywords: polymer extrusion; process monitoring; melt pressure; modelling; process settings; screw geometry; pressure fluctuations; frequency of fluctuations.

Reference to this paper should be made as follows: Abeykoon, C., Li, K., Martin, P.J. and Kelly, A.L. (2012) 'Monitoring and modelling of the effects of process settings and screw geometry on melt pressure generation in polymer extrusion', Int. J. System Control and Information Processing, Vol. 1, No. 1, pp.71-88.

Biographical notes: Currently, Chamil Abeykoon is a Lecturer in mechanical engineering at the University of Peradeniya (Sri Lanka) where he received his BSc in 2007. He obtained his $\mathrm{PhD}$ in process modelling and control from the Queens University Belfast (UK) in 2011 with 13 research publications and a young author best paper award from the IEEE. His research interests include process monitoring, modelling and control; soft sensors and soft sensing; and heat transfer. He is an associate member of the IESL, IMechE and ICPM.

Kang Li received the B.Sc. degree from Xiangtan University in 1989, the M.Sc. degree from the Harbin Institute of Technology in 1992, and the Ph.D. degree in control theory and applications from Shanghai Jiaotong University in 1995, all in China. He is currently a Chair Professor of Intelligent Systems and Control at the School of Electronics, Electrical Engineering and Computer Science, Queen's University Belfast, UK. His research interests covers nonlinear system modelling, identification and control, and bio-inspired computational intelligence, with applications to power systems, polymer extrusion, bioinformatics and systems biology. He has published over 160 papers in his field.

Peter Martin MEng PhD is a Reader in Polymer Engineering at Queen's University Belfast in Northern Ireland and a Fellow of the Institution of Mechanical Engineers (IMechE). He has more than 20 years' experience of research into polymer processes with particular emphasis on extrusion, thermo-forming and blowmoulding. His current research interests include viscoelastic/plastic modelling of polymers, finite element simulation of processes, large strain biaxial testing, inprocess measurement and control, micro-forming and thermoplastic composites.

Adrian Kelly is a Senior Lecturer in Polymer Engineering at the University of Bradford (UK). He is a Chartered Mechanical Engineer and has a BEng in Mechanical Engineering. Adrian was awarded a $\mathrm{PhD}$ in 1997 for research into process metrology of polymer extrusion compounding. His current research interests include extrusion, process monitoring, rheology, pharmaceutical polymer engineering, biopolymers and recycling.

\section{Introduction}

Polymers, because of their range of properties and ease of processing into complex shapes, are among the most important materials available to us today and the polymer industry makes a major contribution to the economy of many countries. Being a fundamental 
method of processing polymer materials, extrusion is becoming increasingly important as a production method in the present industry. In general, it is involved in the final production of many polymer products such as pipes, films, sheets, tubes, rods, etc. It is also used as an intermediate processing stage for injection moulded, thermoformed and blow-moulded products. More details on the basic process mechanisms and operation of polymer extrusion can be found in Rauwendaal (2001) and Stevens and Covas (1995). Usually, the information provided by process parameters such as melt pressure, melt temperature, mass throughput, etc., are taken as the key measures of process stability in polymer extrusion. Of these process parameters, melt pressure is an important characteristic of single screw extruders and strongly influences the process output (Lindt, 1981). In the majority of polymer processes, it is common to measure melt pressure at the end of the extruder barrel and/or at the die, while some of the processes require multiple pressure measurements. Generally, melt pressure information helps to provide process understanding and is highly useful in troubleshooting (Rauwendaal, 2002b). Moreover, melt pressure is dependent on process settings, machine geometry and material properties (Potente and Hanhart, 1994). Therefore, investigation of the effects of process settings on melt pressure development is important for a given screw geometry and polymer material. Attempts made previously on modelling of melt pressure in single screw extrusion are discussed in the following sections.

Donovan (1971) made an analytical approach to predict the pressure profile in the melting (or compression) and metering zones of a single screw extruder. It was found that the pressure profile is highly dependent on the solid bed of polymer under stable melting conditions. Lovegrove and Williams (1974) proposed a theoretical analysis to predict pressure generation in the solids conveying zone of single screw extruders and emphasised the importance of considering screw weight and centrifugal forces for pressure calculations. Lindt (1981) found that increasing throughput tended to decrease the pressure build up capacity of the melting zone in a single screw extruder. Moreover, a relationship between the melting mechanism and pressure profile was discussed. Ghoreishy and Nouri (1999) developed a mathematical model for a 3-D analysis of the melt flow through the metering and die sections of a single screw extruder by using a finite element technique. Generalised Newtonian flow conditions were assumed and the experimentally measured mass flow rate and pressure profile showed good agreement with the simulation results. Work presented by Potente and Jungemann (2000) developed polynomial formulae to describe the correlation between the axial pressure profile and mass throughput along the melting zone of a single screw extruder, which can be used as simulation program models. The proposed models are only applicable for non-Newtonian polymer melts and the authors claimed that good agreement was achieved with the numerical results tested.

Rauwendaal (1986) developed a formula to describe the process mass throughput as a function of the pressure gradient for power law fluids in single screw extruders and mentioned that the expression was well matched with numerical calculations carried out. Furthermore, he argued that when extruder die pressure changes with time, the process output may vary and hence dimensional instability of the products can be caused (Rauwendaal, 2001). Another study by Rauwendaal (2002a) developed a relationship between the extruder output and pressure and found that at low Melt Flow Index (MFI) values, the pressure sensitivity of the output increased as the output reduced. Moreover, a comprehensive analysis of extrusion melt pressure was provided and equations were 
proposed to calculate the axial and circumferential pressure profiles of an extruder and the usefulness of process information given by these pressure profiles was also highlighted (Rauwendaal, 2002b).

Work presented by Tadmor et al. (1974) considered the transport momentum in the solid bed, melt film and melt pool of a single screw extruder. A model was developed to represent the dynamic pressure profile through simulations. A number of assumptions were made and the model involved complex differential equations requiring powerful computer solutions. As a result, it was limited in practical applications. Costin et al. (1982) used time series analysis to develop dynamic transfer function models between the screw speed and pressure and also to model the disturbances associated with the extruder pressure. These models were used in the development of a process control strategy. Kochhar and Parnaby (1977) proposed two time series models of melt pressure and temperature. Linearised models relating to small perturbations of the screw speed were identified by observing corresponding changes of the melt pressure and the temperature at the die and the model was used in formulating a process control framework. Other work was reported on the modelling of the extruder melt pressure for the development of process control strategies (Fontaine, 1975; Parnaby et al., 1975; Patterson and Kerf, 1978; Hassan and Parnaby, 1981; Nelson et al., 1986; Chan et al., 1986) and also for the process identification/monitoring purposes (Caldwell, 2005; McAfee and McNally, 2006).

Tan et al. (2004) proposed a grey-box model of polymer extrusion and stated that the model can be used to estimate melt temperature and pressure at the die. The model was verified through simulation by introducing step changes to the manipulated parameters such as the screw speed. It was reported that the model adapted to the changes of processing conditions and feed materials. Botten et al. (2003) proposed a theoretical model to predict the pressure development in a single screw extruder as a function of machine geometry (i.e., barrel diameter, screw helix angle and channel width), material properties (i.e., yield and shear stresses), screw speed and volumetric flow rate. Experimental work is underway to verify the model performance. Bereaux et al. (2009) introduced a model to predict the throughput and pressure development in single screw extruders. Model predictions were compared with some of the experimental results available in the published literature and the authors claimed that good agreement was achieved. It was found that the throughput is primarily dependent on the screw geometry, while the peak pressure is highly dependent on polymer viscosity. Moreover, they mentioned that the model is applicable to both single screw extrusion and injection moulding. Also, they argue that the level of back pressure (i.e., pressure close to the die entry) in polymer extrusion is dependent upon the screw and die geometries.

From the literature, it is clear that little reported work is available on modelling melt pressure in polymer extrusion based on system identification experiments (i.e., empirical models). The majority of the existing empirical models are based on either the Laplace transfer function or time series techniques. Conversely, the practical use of most of the existing models has been hampered due to problems such as atypical processing conditions used for experiments, limitation to a very narrow processing window, difficulties in real-time measurement of the required process parameters, etc. The existing theoretical models may not provide the actual process dynamics due to a number of simplifying assumptions. Obviously, pressure fluctuations may cause problems in product quality and the fluctuations indicated by the process melt pressure signal provide a good indication of process functional quality. Proper observation of the melt pressure behaviour of the 
process is essential otherwise excessive pressure generation may lead to catastrophic failures (Rauwendaal, 2001). Therefore, understanding of the effects of process settings (i.e., screw speed and barrel set temperatures), materials and machine geometry on pressure development is highly important to maintain the process stability while ensuring process safety.

In this work, non-linear static and linear dynamic models were proposed to predict the extruder melt pressure development (i.e., based on the major process variables) with two different screw geometries. The experiments were carried under realistic processing conditions and melt pressure data were recorded across the full operating speed range of the extruder. Moreover, the barrel set temperatures were selected to cover a broad processing range of the material. The effects on melt pressure development of each of the major process parameters and screw geometry were also discussed by using the models developed together with a frequency analysis of the measured signals. This study was focused on a single screw extruder with one polymer. This paper is a fully extended version of the original one presented in ICMIC 2011 (Abeykoon et al., 2011a).

\section{Equipment and procedure}

All measurements were carried out on a $63.5 \mathrm{~mm}$ diameter (D) single screw extruder (Davis Standard BC-60). A Barrier Flighted (BF) screw with a spiral Maddock mixer (a general purpose screw with a 2.5:1 compression ratio) and a tapered Gradual Compression (GC) screw (with 3:1 compression ratio) were used to process the material. More details of the screws are shown in Figure 1 with the Screw Channel Depths (SCD) of the solids conveying (or feeding) and metering zones. From here onwards, these two screws are denoted as the BF screw and the GC screw, respectively.

Figure 1 Details of the screws: (a) The GC screw and (b) The BF screw

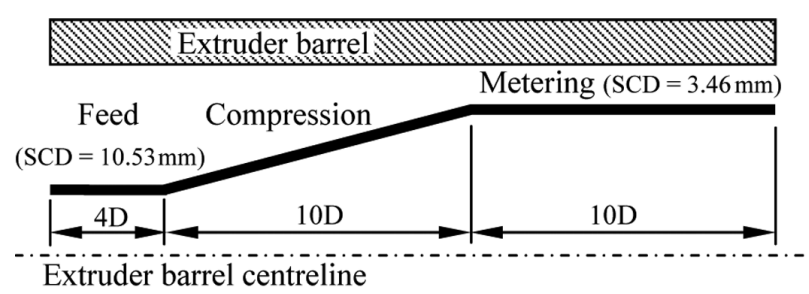

(a)

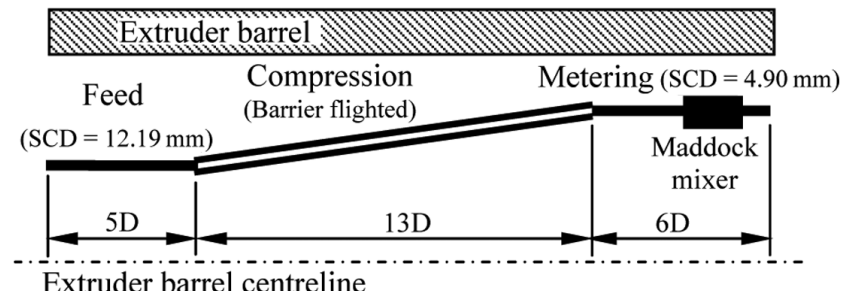

(b)

The extruder was fitted with a $38 \mathrm{~mm}$ diameter adapter by using a clamp ring prior to a short $6 \mathrm{~mm}$ diameter rod die as shown in Figure 2. 
Figure 2 Extruder barrel, adapter, die and melt pressure sensor

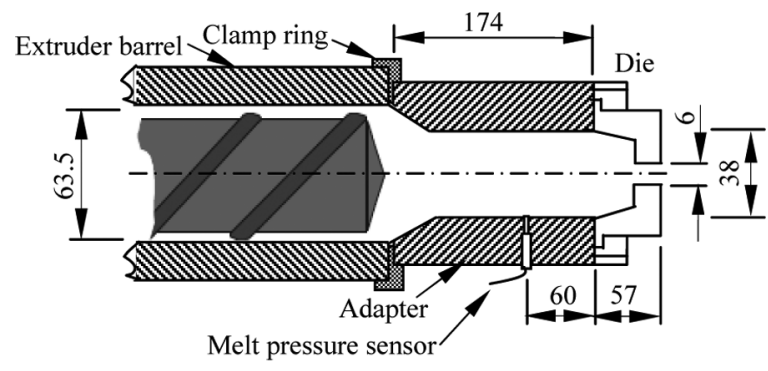

All dimensions are in millimetres

The extruder barrel has four separate temperature zones and another three separate temperature zones at the clamp ring, adapter and die. Each of these temperature zones is equipped with a separate temperature controller that allows individual control of the set temperature. The extruder barrel dimensions and the arrangement of the heaters are shown in Figure 3.

Figure 3 Arrangement of the BC-60 extruder barrel and heaters

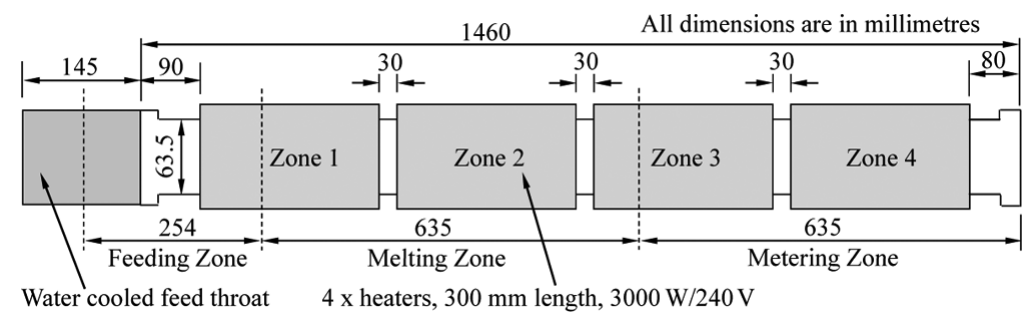

Melt pressure at the adapter (i.e., close to the die entry) was measured using a PT422A Dynisco pressure sensor as shown in Figure 2. A data acquisition programme developed in LabVIEW was used to communicate between the experimental instruments and a PC. The screw speed, barrel temperatures and all melt pressure signals were acquired at $10 \mathrm{~Hz}$ using a 16-bit DAQ card (National Instruments (NI) PCI-6035E) through a NI low-noise SCXI-1000 connector box.

Experimental trials were carried out on a virgin highdensity polyethylene (HDPE), (Exxonmobil HYA 800), (density: $0.961 \mathrm{~g} / \mathrm{cm}^{3}$, MFI: $0.7 \mathrm{~g} / 10 \mathrm{~min} @\left(190^{\circ} \mathrm{C}, 2.16 \mathrm{~kg}\right)$ ). The extruder temperature settings were fixed as described in Table 1 under three different barrel set temperature conditions and denoted as A (high temperature), B (medium temperature) and $\mathrm{C}$ (low temperature).

Table 1 Extruder barrel temperature settings

\begin{tabular}{|c|c|c|c|c|c|c|c|}
\hline \multirow{3}{*}{ 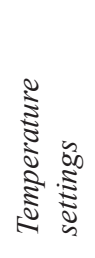 } & \multicolumn{7}{|c|}{ Set temperatures $\left({ }^{\circ} \mathrm{C}\right)$} \\
\hline & \multicolumn{4}{|c|}{ Barrel zones } & \multirow[t]{2}{*}{ 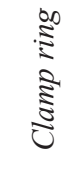 } & \multirow{2}{*}{$\frac{\bar{d}}{\frac{d}{3}}$} & \multirow{2}{*}{ 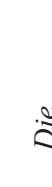 } \\
\hline & 1 & 2 & 3 & 4 & & & \\
\hline A & 110 & 130 & 180 & 230 & 230 & 230 & 230 \\
\hline B & 105 & 125 & 175 & 215 & 215 & 215 & 215 \\
\hline $\mathrm{C}$ & 100 & 120 & 170 & 200 & 200 & 200 & 200 \\
\hline
\end{tabular}


The experiments were started with temperature setting A and data were recorded with the screw stationary for 1 minute. Then, the screw speed was increased up to $90 \mathrm{rpm}$ with random steps of between \pm 5 and $40 \mathrm{rpm}$ and for the different barrel set temperatures with the extruder running for about 151 and 193 minutes continuously in the case of the GC screw and BF screw, respectively. The extruder was allowed to stabilise for 15 minutes after each set temperature change, whereas it was hold for about 7 minutes at each of the other different conditions. All of these settings were selected to generate realistic processing conditions whilst covering the full operating range of the extruder (i.e., $0-100 \mathrm{rpm}$ ). Moreover, the barrel set temperatures were selected to cover a wide processing range of the material while ensuring the normal processing conditions throughout the experiment (i.e., without having problems such as in melting, conveying, etc). Separate tests were carried out for model training and validation and process setting metrics corresponding to the GC and BF screws are shown in Figures 5 and 6 , respectively.

\section{Modelling}

In general, the melt pressure development $(P)$ in polymer extrusion can be represented as a function of $\omega_{s c}$ and $T_{b}$

$$
P=f\left(\omega_{s c}, T_{b}\right),
$$

where $\omega_{s c}$ is the screw speed and $T_{b}$ represents the barrel set temperatures (subscript $b$ represents the different barrel zones $\left.T_{1}-T_{4}\right)$. Five model inputs $\left(\omega_{\mathrm{sc}}, T_{1}, T_{2}, T_{3}\right.$ and $\left.T_{4}\right)$ and one output $(P)$ were considered for the modelling of melt pressure generated as a function of process speed and barrel set temperatures. The selected model structure is illustrated in Figure 4.

Figure 4 Model structure with the selected inputs and output

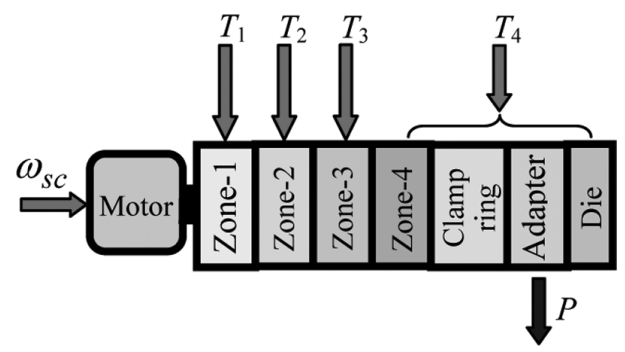

The set temperatures of the clamp ring, the adapter and the die were always equal to $T_{4}$ in this study. If these values vary from $T_{4}$, it is possible to add them as three different model inputs. There are 19 and 25 different processing situations and each input signal used for the model training contains 90,600 and 115,800 data points for the GC and the BF screws, respectively. The model input matrices for both training and validation data are shown in Figures 5 and 6 for the two screws. The model output contains the measured melt pressure at each processing condition corresponding to the relevant inputs and the signal length is the same as the input signal length.

In this work, a linear-in-the-parameters (LITP) modelling technique was used to model the extrusion process. In general, a LITP model is a linear combination of model 
Figure 5 Model input matrices - GC screw (see online version for colours)

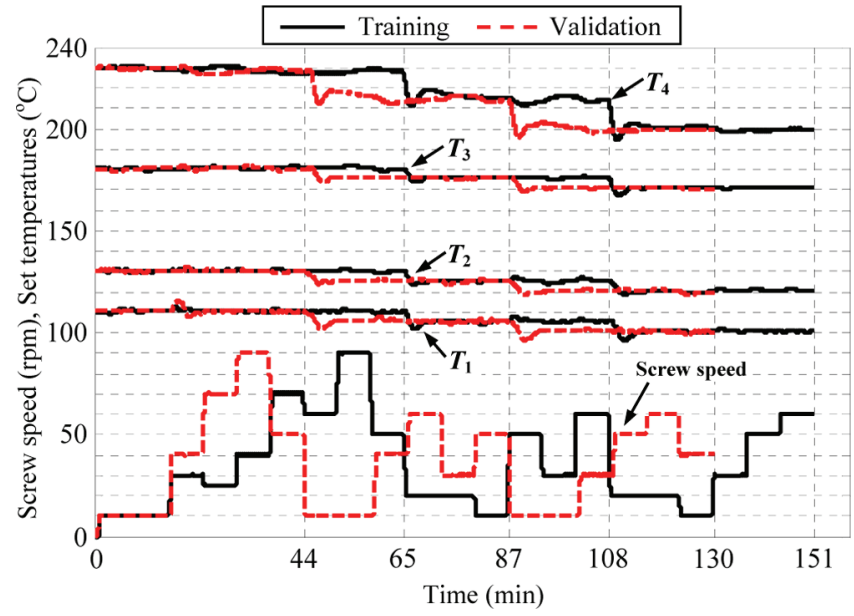

Figure 6 Model input matrices - BF screw (see online version for colours)

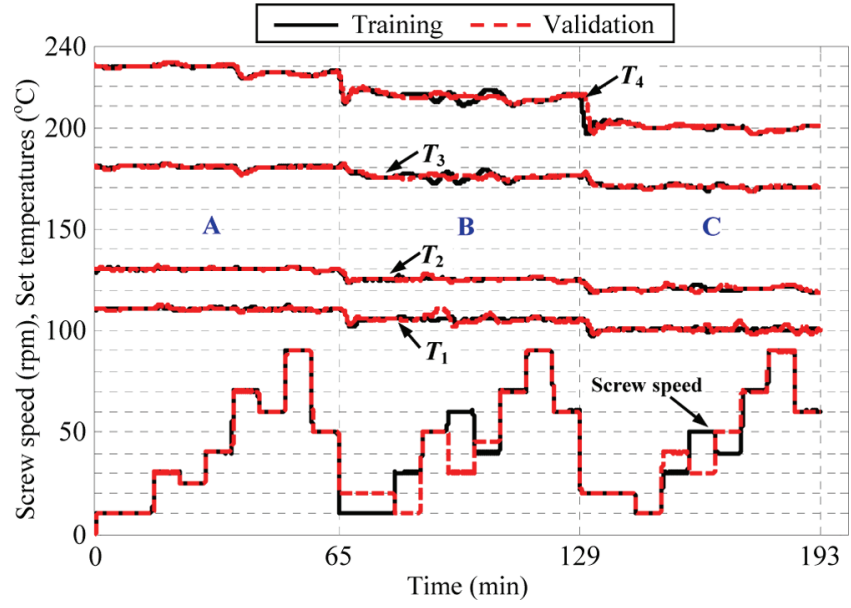

terms, which are linear or non-linear functions of the corresponding system variables and it gives the target output as a linear combination of model terms, the coefficient of each model term can be estimated by linear optimisation methods, such as least squares. This is a common way to build a non-linear system model as the linear systems are too simple to approximate the non-linear characteristics and traditional non-linear systems are difficult to optimise (Li et al., 2005, 2006). Generally, most of the non-linear optimisation methods require extensive computation and a large amount of memory and the resulting model is usually a sub-optimal. Therefore, the LITP model is a compromise of the above two construction schemes as it does not require huge computational power for structure and parameter estimations. Due to their ability for universal approximation and simple structure, LITP models have been widely used in a number of practical applications such as signal processing, data mining, pattern recognition, time series prediction and non-linear 
system modelling and identification etc., (Zhu and Billings, 1996; Hong et al., 2008; Li et al., 2009; Liu et al., 2011).

A two-stage algorithm ( $\mathrm{Li}$ et al., 2005, 2006) was employed in the selection and refinement of the LITP models for this study. In the first stage, a Fast Recursive Algorithm (FRA) was used for the selection of the model structure and for estimation of the model parameters. This solves the problem recursively and does not require matrix decomposition as is the case for Orthogonal Least Squares (OLS) techniques (Chen et al., 1989). However, the models developed include a constraint that the terms added later are based on previously selected ones. As a result, some of them may not have a significant contribution. Then, in the second stage, a backward model refinement procedure was carried out to eliminate non-significant terms to build up a compact model. The significance of each selected model term was reviewed and compared with those remaining in the candidate term pool and all insignificant terms were replaced, leading to improved performance without increasing the model size. The authors have used the same modelling technique for the modelling of the die melt temperature profile (Abeykoon et al., 2010a, 2011c,b) and motor power consumption (Abeykoon et al., 2010c) in polymer extrusion and good results have been achieved.

\section{Results and discussion}

Firstly, linear models were adopted to approximate the system under static and dynamic conditions. However, performance of the linear models under static conditions was poorer and hence, static non-linear models were developed. Linear dynamic models were able to provide good performance in predicting melt pressure development for both screw geometries. Therefore, the static non-linear and dynamic linear models are discussed in detail. To test the dynamic model accuracy, the Modelling Errors (ME) and the Root Mean Square Errors (RMSE) of the models were determined by equations (2) and (3), respectively.

$$
\begin{aligned}
& M E=y_{i}(t)-\hat{y}_{i}(t) \\
& R M S E=\sqrt{\frac{1}{N} \sum_{i=1}^{N}\left[\left(\hat{y}_{i}(t)-y_{i}(t)\right)\right]^{2}}
\end{aligned}
$$

where $y_{i}(t)$ is the measured melt pressure at time $t, \hat{y}_{i}(t)$ is the model estimated melt pressure at time $t$ and $N$ is the number of data points. The same equations without the involvement of time were used to test the accuracy of the static models.

\subsection{Linear dynamic models}

For the selection of the linear dynamic melt pressure prediction model, a number of different model combinations (i.e., models with different orders and number of terms) were studied. Two past output terms and one past input term from each input were used to predict the current output (i.e., $n_{a}=2$ and $n_{b}$ for each input is equal to 1 ). Then, the maximum delays $\left(n_{k}\right)$ attributed to each model input had to be determined. Therefore, melt pressure changes followed by screw speed changes and barrel set temperature changes were observed from the experimentally measured data. The process pressure changes immediately after any 
change of screw speed. Also, the melt pressure is affected by barrel set temperatures, but it takes a slightly longer period of time to change the barrel zone temperatures once any change is made. Based on these observations, reasonable values were assumed for the delays attributed to each input as: $d-\omega_{\mathrm{sc}}=10 \mathrm{~s}, d-T_{1}=120 \mathrm{~s}, d-T_{2}=120 \mathrm{~s}, d-T_{3}=120 \mathrm{~s}$ and $d-T_{4}=120 \mathrm{~s}$. These delays can be adjusted as required depending on the screw geometry, material, processing conditions, etc. The details of some of the dynamic models studied are shown in Table 2 along with their RMSE when compared with the unseen data for both screws.

Table 2 RMSE (on the unseen data) of the studied dynamic models with different orders and number of terms

\begin{tabular}{|c|c|c|c|c|c|c|}
\hline \multirow{3}{*}{ 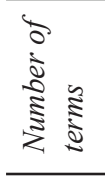 } & \multicolumn{6}{|c|}{ RMSE with the selected model order } \\
\hline & \multicolumn{3}{|c|}{ GC screw } & \multicolumn{3}{|c|}{ BF screw } \\
\hline & 1 & 2 & 3 & 1 & 2 & 3 \\
\hline 1 & 0.0127 & 0.0127 & 0.0127 & 0.0333 & 0.0333 & 0.0333 \\
\hline 2 & 0.0126 & 0.0126 & 0.0126 & 0.0328 & 0.0328 & 0.0328 \\
\hline 3 & 0.0126 & 0.0126 & 0.0126 & 0.0328 & 0.0328 & 0.0328 \\
\hline 4 & 0.0126 & 0.0126 & 0.0126 & 0.0328 & 0.0328 & 0.0328 \\
\hline 5 & 0.0126 & 0.0126 & 0.0126 & 0.0328 & 0.0322 & 0.0322 \\
\hline 6 & 0.0126 & 0.0129 & 0.0129 & 0.0328 & 0.0316 & 0.0317 \\
\hline 7 & 0.0126 & 0.0133 & 0.0133 & 0.0328 & 0.0316 & 0.0314 \\
\hline 8 & 0.0126 & 0.0133 & 0.0133 & 0.0328 & 0.0311 & 0.0314 \\
\hline
\end{tabular}

From the linear dynamic models studied, models with seven terms were selected for further analysis and are shown in equations (4) and (5) for the GC and BF screws, respectively. These models show RMSE of 0.013 and 0.033 on the unseen data, respectively.

$$
\begin{aligned}
\hat{P}_{G C}(t) & =0.65331 \times \hat{P}_{G C}(t-1)+0.34513 \times \hat{P}_{G C}(t-2) \\
& +0.00031 \times T_{3}(t-120)-0.00019 \times T_{2}(t-120) \\
& +0.00013 \times \omega_{S C}(t-10)-0.00010 \times T_{4}(t-120) \\
& -6.18332 \times 10^{-05} \times T_{1}(t-120) \\
\hat{P}_{B F}(t) & =0.89464 \times \hat{P}_{B F}(t-1)+0.10459 \times \hat{P}_{B F}(t-2) \\
& -0.00022 \times T_{2}(t-120)-7.43988 \times 10^{-05} \times T_{1}(t-120) \\
& +0.00014 \times T_{3}(t-120)+5.38979 \times 10^{-05} \times T_{4}(t-120) \\
& +4.69300 \times 10^{-05} \times \omega_{S C}(t-10)
\end{aligned}
$$

The prediction ability of the selected dynamic models on the unseen data is shown in Figure 7 for both screws along with their model prediction errors. 
Figure 7 Performance of the selected linear dynamic models on the unseen data with the Model Prediction Error (ME): (a) GC screw and (b) BF screw (see online version for colours)
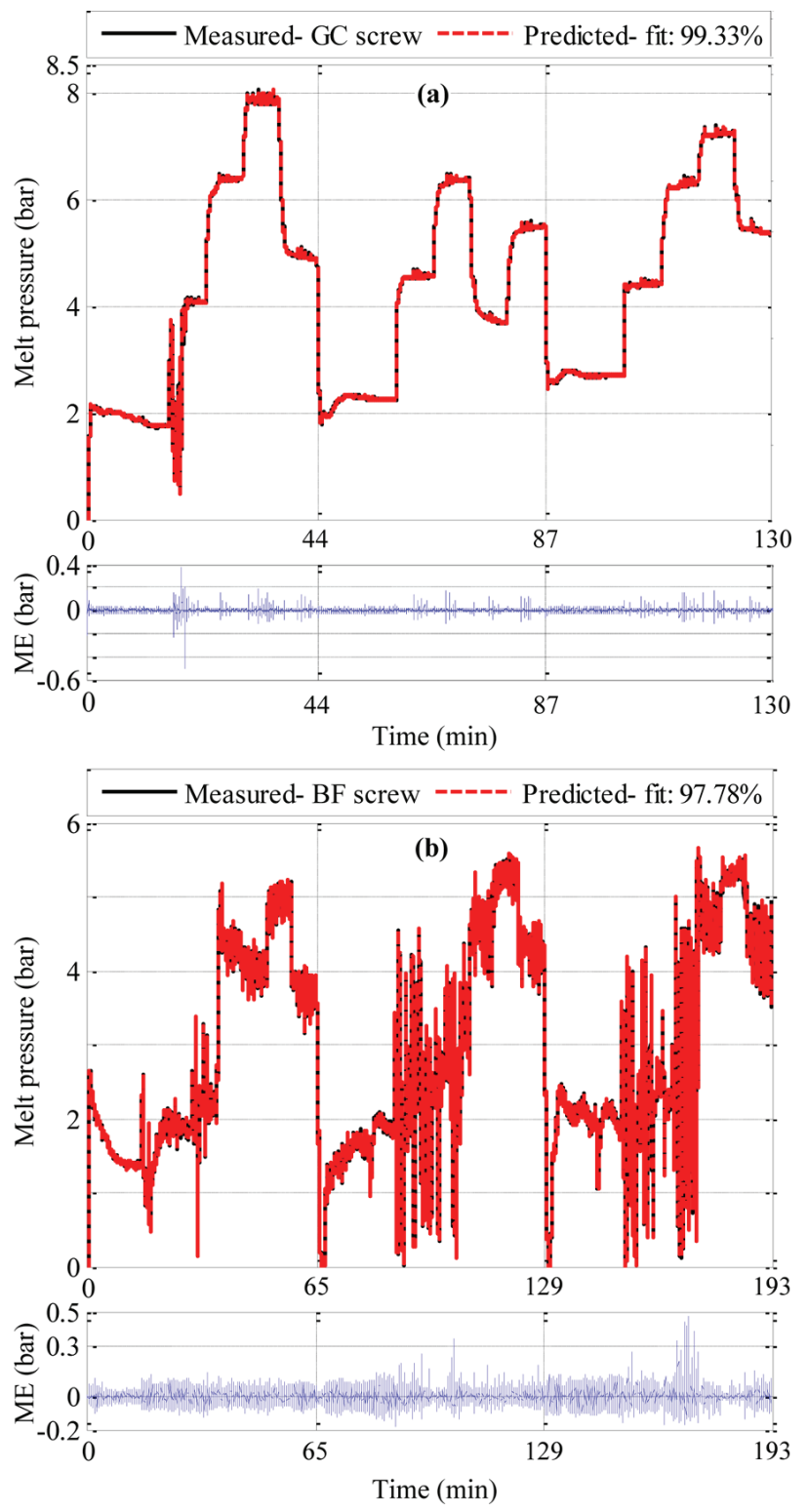

In general, both models show good prediction accuracy on the unseen data over all the processing conditions.

\subsection{Non-linear static models}

For the selection of static models, different model combinations were also studied and details are given in Table 3 along with their RMSE on the unseen data for both screws. 
Table 3 RMSE (on the unseen data) of the studied static models with different orders and number of terms

\begin{tabular}{|c|c|c|c|c|c|c|}
\hline \multirow{3}{*}{ 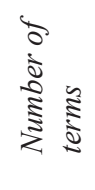 } & \multicolumn{6}{|c|}{ RMSE with the selected model order } \\
\hline & \multicolumn{3}{|c|}{ GC screw } & \multicolumn{3}{|c|}{ BF screw } \\
\hline & 1 & 2 & 3 & 1 & 2 & 3 \\
\hline 1 & 1.0701 & 1.0701 & 1.0701 & 0.8883 & 0.8883 & 0.8883 \\
\hline 2 & 0.0688 & 0.7928 & 0.7885 & 0.8092 & 0.8092 & 0.8092 \\
\hline 3 & 0.4170 & 0.4224 & 0.4240 & 0.8099 & 0.8038 & 0.8117 \\
\hline 4 & 0.3967 & 0.4135 & 0.3095 & 0.8117 & 0.7776 & 0.7917 \\
\hline 5 & 0.3996 & 0.4112 & 0.4029 & 0.8137 & 0.7737 & 0.8009 \\
\hline 6 & 0.3996 & 0.4069 & 0.3990 & 0.8137 & 0.7812 & 0.7783 \\
\hline 7 & 0.3996 & 0.4079 & 0.4045 & 0.8137 & 0.7761 & 0.7679 \\
\hline 8 & 0.3996 & 0.4129 & 0.4057 & 0.8137 & 0.7752 & 0.7630 \\
\hline 9 & 0.3996 & 0.4087 & 0.4121 & 0.8137 & 0.7734 & 0.7552 \\
\hline 10 & 0.3996 & 0.4095 & 0.4087 & 0.8137 & 0.7733 & 0.7617 \\
\hline 11 & 0.3996 & 0.4238 & 0.4145 & 0.8137 & 0.7899 & 0.7520 \\
\hline 12 & 0.3996 & 0.3981 & 0.4258 & 0.8137 & 0.7908 & 0.7551 \\
\hline 13 & 0.3996 & 0.4098 & 0.4049 & 0.8137 & 0.7926 & 0.7556 \\
\hline 14 & 0.3996 & 0.4036 & 0.4279 & 0.8137 & 0.7922 & 0.7560 \\
\hline
\end{tabular}

Finally, second-order static non-linear models with 12 terms were selected for discussion and are given in equations (6) and (7) for the GC and BF screws, respectively.

$$
\begin{aligned}
\hat{P}_{G C}= & -0.42177 \times \omega_{S C}+0.21133 \times T_{3}-0.05896 \times T_{2} \times T_{3} \\
& +0.03528 \times T_{2}^{2}+0.00075 \times T_{1} \times T_{4}+0.00920 \times \omega_{S C} \times T_{3} \\
& -0.00237 \times T_{4}^{2}-0.00498 \times \omega_{S C} \times T_{1}-0.00458 \times \omega_{S C} \times T_{2} \\
& -0.00014 \times \omega_{S C}^{2}+0.00733 \times T_{2} \times T_{4}+0.01953 \times T_{3}^{2} \\
\hat{P}_{B F}= & +2.70469 \times T_{3}-1.43535 \times T_{4}-1.33292 \times T_{2} \\
& +0.89517 \times \omega_{S C}+0.09102 \times T_{2} \times T_{3}-0.08794 \times T_{2}^{2} \\
& -0.3979 \times T_{3}^{2}+0.03357 \times T_{2} \times T_{4}-0.00887 \times \omega_{S C} \times T_{3} \\
& -0.00643 \times T_{4}^{2}+0.00312 \times \omega_{S C} \times T_{4}+0.00052 \times \omega_{S C}^{2}
\end{aligned}
$$

These static polynomial models of the GC and BF screws show 0.398 and 0.791 RMSE on the unseen data, respectively.

\subsection{Effects of process settings and screw geometry on melt pressure}

As described in Section 2, both experiments were carried out on the same material under the same processing conditions (i.e., the same barrel set temperatures). As evidenced by Figure 7, relatively stable pressure generation can be seen with the GC screw, whereas experiments with the BF screw show pressure fluctuations over some screw speeds. According to Gitschner and Lutterback (1984), process pressure variations are inter-related 
with the barrel zone temperatures and process cooling systems and it is possible to stabilise pressure surges by adjusting these parameters. In this study, it was felt that fluctuations observed with the BF screw were as a result of the incompatibility of the selected barrel set temperatures with the screw geometry for this particular material. It was very difficult to select suitable barrel set temperatures for the HDPE material used as the material tended to slip on the screw. As a result, material conveying was poor and gave very low process pressures and hence reduced throughput rates. Therefore, the barrel set temperatures shown in Table 1 were selected after long periods of adjustments, particularly in the first two barrel zones (i.e., $T_{1}$ and $T_{2}$ ) to avoid conveying problems. These adjustments were only carried out with the GC screw and then the experiments with the BF screw were carried out with the same set temperatures. This may be the reason for the higher pressure fluctuations with the BF screw than the GC screw as shown in Figure 7. Usually, BF screws perform favourably (e.g., efficient melting and mixing) compared with conventional GC screws (Brown et al., 2004; Kelly et al., 2006). Therefore, the results in this study support the argument made by Gitschner that barrel set temperatures have to be changed from screw to screw although the machine and processing material remain the same. However, this material slipping condition may depend on the frictional and shear forces between the metal surfaces (i.e., barrel and screw) and the pellets (i.e., material) and also on the pellet size and shape (Rauwendaal, 2001; Chung et al., 1977). Achieving proper material conveying (i.e., a proper axial pressure profile) along all screw zones is a major requirement to achieve stable pressure generation and this ensures a uniform process output (Rauwendaal, 2002b). Therefore, appropriate set temperatures should be selected for all barrel temperature zones to avoid possible conveying problems, which may lead to pressure fluctuations.

In general, it can be considered that these experiments were carried out under proper material conveying conditions. Therefore, the models developed can be used to identify the effects of process settings and screw geometry on melt pressure development. As shown in Figure 7, the process pressure developed is higher with the GC screw than the BF screw, which can be attributed to the geometrical differences between the screws. According to the static models, screw speed is the most significant process parameter for the GC screw, while the third barrel zone temperature $\left(T_{3}\right)$ is the most significant with the BF screw. Of the barrel zone temperatures, the zone 3 temperature seems to be the most significant for both experiments under static processing conditions despite the differences in screw geometry. As shown in Figure 3, $T_{3}$ pertains both to the melting and metering zones of the extruder barrel. In the dynamic models, all barrel set temperatures and screw speed show low coefficients. Overall, all static and dynamic models show the significance of the temperatures of the end barrel zones (i.e., $T_{2}, T_{3}$ and $T_{4}$ ) on the melt pressure development where molten material exists. Therefore, under proper conveying conditions, the significance of the feed (or solids conveying) zone temperature $\left(T_{1}\right)$ on the process melt pressure seems to be negligible.

\subsection{Frequency analysis of the measured melt pressure signals}

For further understanding of the process melt pressure behaviour, an orthonormal Discrete Wavelet Transform (DWT) analysis of the measured melt pressure signals with both the GC and BF screws (i.e., the signals of the validation tests shown in Fig. 7) were carried out based on the Daubechies (db) method (Daubechies, 1992). In general, DWT performs signal analysis by decomposing the signal into different frequency bands, such that the variations (i.e., magnitude of fluctuations) within each frequency band against time can be observed. 
For this study, a db20 method was used and the signals were decomposed into 10 frequency bands. The frequencies relevant to each frequency band of the reconstructed orthogonal signals are given in Table 4.

Table 4 Frequency ranges of each level

\begin{tabular}{lccc}
\hline Levels & Frequency range $(\mathrm{Hz})$ & Levels & Frequency range $(\mathrm{Hz})$ \\
\hline 10 & $0.005-0.010$ & 5 & $0.156-0.312$ \\
9 & $0.010-0.020$ & 4 & $0.312-0.625$ \\
8 & $0.020-0.039$ & 3 & $0.625-1.250$ \\
7 & $0.039-0.078$ & 2 & $1.250-2.500$ \\
6 & $0.078-0.156$ & 1 & $2.500-5.000$ \\
\hline
\end{tabular}

The wavelet frequency plots of the measured pressure signals with the GC and BF screws are shown in Figures 8(a) and 8(b), respectively. As was evidenced from Figure 7, pressure

Figure 8 Wavelet frequency of the measured melt pressure signals: (a) with the GC screw (b) with the BF screw (see online version for colours)

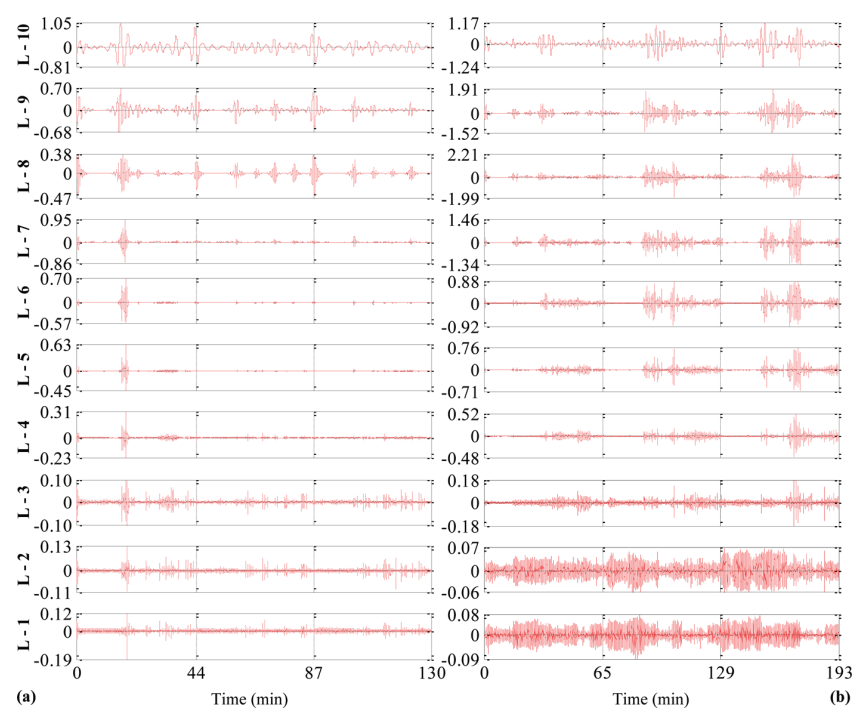

fluctuations are higher with the BF screw than the GC screw and this is reflected by the wavelet frequency plots as well (i.e., the magnitudes of the frequency plots of the BF screw in levels 10-3 are higher than the GC screw). The highest magnitude of fluctuations with the GC screw is shown at level 10, while level 8 is the highest with the BF screw. After 16 minutes of process operation (i.e., after applying the screw speed step change from 10 to $40 \mathrm{rpm}$ under the set temperature condition $\mathrm{A}$ ), the process melt pressure does not show significant fluctuations over $0.039 \mathrm{~Hz}$ with the BF screw although a number of process setting changes were applied after this time period. However, this is not the case with the BF screw although the same processing conditions and material were used with both screws. In general, neither of the screws does show significant pressure fluctuations higher than $0.625 \mathrm{~Hz}$ (i.e., the 
magnitudes of the wavelet plots are below 0.5 in the frequency levels 3-1 for both screws). Conversely, these frequency plots provide an indication of the effects of the process settings (i.e., screw speed and barrel set temperatures) on the process pressure fluctuations. As is clear from Figure 8(b), the BF screw showed an increase in pressure fluctuations as the barrel set temperatures decreased from condition A to C. Moreover, the melt pressure of the BF screw showed a trend of increasing fluctuations with screw speed. However, a clear relationship between the changes to process settings and pressure fluctuations cannot be observed with the GC screw from the corresponding wavelet frequency plots. Clearly, the results of the frequency analysis are also indicated that the process melt pressure generation is influenced by both the screw geometrical properties and the process settings. Moreover, some of the issues relating to melt pressure fluctuations and process stability were discussed in the previous publications (Abeykoon et al., 2010b, 2011d).

In general, fluctuations observed in the melt pressure signal may be attributed to solids conveying, melting or metering (i.e., melt conveying) problems causing various adverse effects to the product quality. Furthermore, melt pressure fluctuations are directly related to the process melt output fluctuations (Lindt, 1981). Therefore, process operators should have a sound knowledge on the relationships between the machine geometry, process settings and materials for achieving a stable process operation. However, this may be extremely challenging as a large number machines (i.e., with the differences in size, operation, components, etc.) and materials (i.e., with the differences in the type, grade, form, etc.) are used in industry. Also, it seems that there are not many practical quantitative expressions to relate important process parameters and machine geometry and/or materials available in the present industry. Usually, process operators deal with issues such as the selection of the process settings and product quality control by trial and error, which is inefficient and can result in considerable waste of raw materials, labour, energy, time, etc. Therefore, the development of general quantitative expressions between the important process parameters, machine geometry and materials should help to improve the efficiency of polymer processing applications.

\section{Conclusions}

New static and dynamic models have been presented to predict melt pressure in polymer extrusion as a function of readily measurable process parameters. A computationally efficient LITP modelling technique was used and the models developed were able to predict the process melt pressure with good accuracy over a wide operating window. These models were used to identify the effects of individual processing parameters and screw geometry on the process melt pressure generation. The results showed that the process settings should be selected based on the screw geometry for achieving a stable melt pressure condition although the same machine and material are used. Otherwise, problematic conveying issues may occur leading to pressure fluctuations. In general, investigations made by both the models developed and the frequency analysis confirmed that the process melt pressure generation was dependent upon the screw geometry and the processing conditions. Moreover, the significance of each processing parameter on the level of melt pressure seems to be dependent upon the screw geometry. As the predictions of the proposed models were well matched with the experimentally measured pressure traces, they are useful in processing issues such as obtaining optimum process settings to avoid possible melt pressure fluctuations. In fact, this 
study was carried out on a single material with two different screw geometries and one die geometry. Development of models for different materials and die geometries should help to identify the specific effects of material and die geometry on melt pressure development. Furthermore, the melt pressure is not only a function of process settings and consideration of both the effects of material properties, (e.g., thermal conductivity, melt consistency index, etc) and machine geometry, (e.g., screw channel depth, barrel diameter surface conditions of the barrel and screw, barrel thickness) should help to improve the model performance further and will be examined in future work.

\section{References}

Abeykoon, C., Li, K., Martin, P.J. and Kelly, A.L. (2011a) 'Modelling of melt pressure development in polymer extrusion: Effects of process settings and screw geometry', 3rd IEEE International Conference on Modelling, Identification and Control, pp.197-202.

Abeykoon, C., Li, K., McAfee, M., Martin, P.J., Deng, J. and Kelly, A.L. (2010a) 'Modelling the effects of operating conditions on die melt temperature homogeneity in single screw extrusion', UKACC International Conference on Control, pp.42-47.

Abeykoon, C., Li, K., McAfee, M., Martin, P.J. and Irwin, G.W. (2011b) 'Extruder melt temperature control with fuzzy logic', 18th IFAC World Congress, pp.8577-8582.

Abeykoon, C., Li, K., McAfee, M., Martin, P.J., Niu, Q., Kelly, A.L. and Deng, J. (2011c) 'A new model based approach for the prediction and optimisation of thermal homogeneity in single screw extrusion', Control Engineering Practice, Vol. 19, No. 8, pp.862-874.

Abeykoon, C., McAfee, M., Li, K., Kelly, A.L. and Brown, E.C. (2010b) 'Monitoring the effect of operating conditions on melt temperature homogeneity in single-screw extrusion', SPE ANTEC Technical Papers, Vol. 1 pp.1799-1806.

Abeykoon, C., McAfee, M., Li, K., Martin, P.J., Deng, J. and Kelly, A.L. (2010c) 'Modelling the effects of operating conditions on motor power consumption in single screw extrusion', LSMS \& ICSEE 2010 - (Part II, LNCS 6329), pp.9-20.

Abeykoon, C., McAfee, M., Li, K., Martin, P.J. and Kelly, A.L. (2011) 'The inferential monitoring of screw load torque to predict process fluctuations in polymer extrusion', Journal of Materials Processing Technology, Vol. 211, No. 12, pp.1907-1918.

Bereaux, Y., Charmeaub, Y.J. and Moguedet, M. (2009) 'A simple model of throughput and pressure development for single screw', Journal of Materials Processing Technology, Vol. 209, No. 1, pp.611-618.

Botten, A.J., Burbidge, A.S. and Blackburn, S. (2003) 'A model to predict the pressure development in single screw extrusion', Journal of Materials Processing Technology, Vol. 135, Nos. 2-3, pp.284-290.

Brown, E.C., Kelly, A.L. and Coates, P.D. (2004) 'Melt temperature homogeneity in single screw extrusion: effect of material type and screw geometry', SPE ANTEC Technical Papers, Vol. 1 pp.183-187.

Caldwell, L. (2005) Modelling of Polymer Extrusion, PhD. Thesis, Queen’s University Belfast.

Chan, D., Nelson, R.W. and Lee, L.J. (1986) 'Dynamic behaviour of a single screw plasticating extruder part II: Dynamic modelling’, Polymer Engineering and Science, Vol. 26, No. 20, pp.152-161.

Chen, S., Billings, S. and Luo, W. (1989) 'Orthogonal least squares methods and their application to non-linear system identification’, International Journal of Control, Vol. 50, No. 5, pp.1873-1896.

Chung, C.I., Hennessey, W.J. and Tusim, M. (1977) 'Frictional behavior of solid polymers on a metal surface at processing conditions’, Polymer Engineering and Science, Vol. 17, No. 1, pp.9-20.

Costin, M.H., Taylor, P.A. and Wright, J.D. (1982) 'On the dynamics and control of a plasticating extruder', Polymer Engineering and Science, Vol. 22, No. 17, pp.1095-1106. 
Daubechies, I. (1992) Ten Lectures on Wavelets, Wiley, New York.

Donovan, R.C. (1971) 'Pressure profiles in plasticating extruders', Polymer Engineering and Science, Vol. 11, No. 6, pp.484-491.

Fontaine, W. (1975) Thesis, Ohio State University.

Ghoreishy, M.H.R. and Nouri, M.R. (1999) 'Finite element analysis of thermoplastic melts glow through the metering and die regions of single screw extruders', Journal of Applied Polymer Science, Vol. 74, No. 3, pp.676-689.

Gitschner, H.W. and Lutterback, J. (1984) 'Influences of barrel wall temperature on melt throughput fluctuations in the single screw extruder', Kunststoffe, Vol. 74, No. 1, pp.7-8.

Hassan, G.A. and Parnaby, J. (1981) 'Model reference optimal steady-state adaptive computer control of plastics extrusion processes', Polymer Engineering and Science, Vol. 21, No. 5, pp. $276-284$.

Hong, X., Mitchell, R., Chen, S., Harris, C.J., Li, K. and Irwin, G.W. (2008) 'Model selection approaches for non-linear system identification: a review', International Journal of Systems Science, Vol. 39, No. 10, pp.925-946.

Kelly, A.L., Brown, E.C. and Coates, P.D. (2006) 'The effect of screw geometry on melt temperature profile in single screw extrusion', Polymer Engineering and Science, Vol. 46, No. 12, pp.1706-1714.

Kochhar, A. and Parnaby, J. (1977) 'Dynamic modelling and control of plastics extrusion processes', Automatica, Vol. 13, No. 2, pp.177-183.

Li, K., Peng, J. and Bai, E. (2006) 'A two-stage algorithm for identification of nonlinear dynamic systems', Automatica, Vol. 42, No. 7, pp.1189-1197.

Li, K., Peng, J. and Bai, E-W. (2009) 'Two-stage mixed discrete-continuous identification of Radial Basis Function (rbf) neural models for nonlinear systems', IEEE Transactions on Circuits and Systems, Vol. 56, No. 3, pp.630-643.

Li, K., Peng, J. and Irwin, G.W. (2005) 'A fast nonlinear model identification method', IEEE Transactions on Automatic Control, Vol. 50, No. 8, pp.1211-1216.

Lindt, J.T. (1981) 'Pressure development in the melting zone of a single-screw extruder', Polymer Engineering and Science, Vol. 16, No. 4, pp.1162-1166.

Liu, X., Li, K., McAfee, M. and Irwin, G. (2011) 'Improved nonlinear PCA for process monitoring using support vector data description', Journal of Process Control, Vol. 21, No. 9, pp.1306-1317.

Lovegrove, J.G.A. and Williams, J.G. (1974) 'Pressure generation mechanisms in the feed section of screw extruders', Polymer Engineering and Science, Vol. 14, No. 8, pp.584-594.

McAfee, M. and McNally, G. (2006) 'Real-time measurement of melt viscosity in single-screw extrusion', Transactions of the Institute of Measurement and Control, Vol. 28, No. 5, pp.481-497.

Nelson, R.W., Chan, D., Yang, B. and Lee, L.J. (1986) 'Dynamic behaviour of a single screw plasticating extruder part I: Experimental study', Polymer Engineering and Science, Vol. 26, No. 20, pp.144-151.

Parnaby, J., Kochhr, A.K. and Wood, B. (1975) 'Development of computer control strategies for plastic extruders', Polymer Engineering and Science, Vol. 15, No. 8, pp.594-605.

Patterson, I. and Kerf, T.D. (1978) 'The dynamic behaviour of extruders', SPE ANTEC Technical Papers, pp.483-487.

Potente, H. and Hanhart, W. (1994) 'Design and processing optimization of extruder screws', Polymer Engineering and Science, Vol. 34, No. 11, pp.937-945.

Potente, H. and Jungemann, J. (2000) 'Pressure/throughput behaviour in the melting section', Polymer Engineering and Science, Vol. 20, No. 5, pp.329-350.

Rauwendaal, C. (1986) 'Throughput-pressure relationships for power law fluids in single screw extruders', Polymer Engineering and Science, Vol. 26, No. 18, pp. 1240-1244.

Rauwendaal, C. (2001) Polymer Extrusion, 4th Ed., Hanser, Munich.

Rauwendaal, C. (2002a) 'Extruder output pressure-relationships for power law fluids including leakage flow, SPE ANTEC Technical Papers, Vol. 1, Paper No: 770. 
Rauwendaal, C. (2002b) 'Pressure distribution in screw extruders', SPE ANTEC Technical Papers, Vol. 1 pp.10-15.

Stevens, M.J. and Covas, J.A. (1995) Extruder Principles and Operation, 2nd Ed., Chapman \& Hall.

Tadmor, Z., Lipshitz, S.D. and Lavie, R. (1974) 'Dynamic model of a plasticating extruder', Polymer Engineering and Science, Vol. 14, No. 2, pp.112-119.

Tan, L.P., Lotfi, A., Lai, E. and Hull, J.B. (2004) 'Soft computing applications in dynamic model identification of polymer extrusion process', Applied Soft Computing, Vol. 4, No. 4, pp.345-355.

Zhu, Q.M. and Billings, S.A. (1996) 'Fast orthogonal identification of nonlinear stochastic models and radial basis function neural networks', International Journal of Control, Vol. 64, No. 5, pp.871-886. 